\title{
Development of Automatic Intelligent System for On-Line Voltage Security Control of Power Systems
}

\author{
N.Tomin, A.Zhukov \\ V. Kurbatsky, D. Sidorov \\ Dept. of Power Systems \\ Melentiev Energy Systems Institute \\ Irkutsk Russia \\ tomin.nv@gmail.com
}

\author{
M. Negnevitsky \\ School of Engineering and ICT \\ University of Tasmania \\ Hobart, Australia \\ Michael.Negnevitsky@utas.edu.au
}

\begin{abstract}
The majority of recent large-scale blackouts have been caused by voltage instability. A prompt on-line assessment of voltage stability for preventive corrective control of electric power systems is one of the key objectives for Control centers. The use of classical approximation methods alone is complicated. Therefore, several modified methods combined with machine learning algorithms enabling security assessment in real time have been proposed over the last years. The paper presents an automatic intelligent system for on-line voltage security control, which is based on the model of decision trees Proximity Driven Streaming Random Forest (PDSRF). In this case, the combination of original properties of PDSRF and capabilities of L-index as a target vector makes it possible to provide the functions of dispatcher warning, localization of critical nodes, and ensure direct interaction with the security automation systems. The efficiency of the proposed system was demonstrated using various test schemes of IEEE.
\end{abstract}

Index Terms-power system, voltage security, control, random forest, security assessment, $L$-index.

\section{INTRODUCTION}

Analysis of mechanisms of the development of large scale blackouts makes an emphasis on the voltage instability as a main reason for blackouts in electric power systems [1, 2]. Enhancement of the relay protection systems, voltage controllers, synchronous compensators as well as an increase in generator rotor speed led to a rise in the power flow dynamic stability limits of electric power systems, which allowed transmission of large amounts of power at considerable distances. Against the background of the rise in the transfer capability, it becomes necessary to meet the requirements for a necessary level of system security by providing sufficient backup sources of reactive power, which is not always fully implemented. This, in turn facilitates voltage instability, which can lead to voltage collapse in a large-scale power system.

In a general case to assess voltage stability in power system in real time, we state the problem of finding the distance of an operating point from voltage stability. The measure obtained may be qualitative or quantitative. The qualitative measure normally requires less computation time compared to the quantitative. It does not provide an estimate of exact power reserve

\footnotetext{
This work was supported by the Russian Scientific Foundation under Grant No. 14-19-00054
}

in megawatts but determines some dimensionless number (that normally varies from 0 to 1 ), which can be interpreted as a stability indicator.

By definition, such indicators of security are scalar magnitudes and are determined by monitoring of a change in certain parameters of electric power systems. These stability indices seem quite convenient for dispatchers, first of all, to generate respective preventive control actions. According to [3] all voltage stability indices of power systems are divided into two main parts: Jacobian matrix based voltage stability indices and system variables based voltage stability indices.

The first group of indices normally requires considerable computational efforts, which imposes limitations on their application for the real-time security assessment. Much less time is necessary to calculate the second group of indicators. This is associated with the fact that their calculation requires only the elements of the admittance matrix and some system variables such as bus voltages or power flow through the lines. Both types of indices vary in the range from 0 to 1 where 0 is no-load and 1 - voltage collapse.

In the traditional statement, the application of such stability indices implies a purely algorithmic approach where the specified equations or partial derivatives are used to calculate numerical values of these indices for each current state of power system. However, as the practice of operation shows, such an approach has a number of significant downsides. These are: low robustness to erroneous inputs; computational complexity erroneous identification of states, and some others [4].

One of the effective solutions to this problem is the use of a combination of traditional approaches on the basis of voltage stability indices and machine learning algorithms. The main idea here lies in an intelligent model learning to independently determine current value of an assumed indicator on the basis of input data, thus identifying the current state of power system. As the studies [5,6] show such a modified approach makes it possible to neutralize the drawbacks of traditional algorithmic approaches owing to the original properties of the machine learning technologies.

The authors tested the most popular machine learning models such as neural networks, support vector machines, various 
decision tree algorithms that have been proposed recently to solve the problems of security assessment of electric power systems [6-8]. Capability of these algorithms to effectively identify operating conditions of electric power systems was demonstrated on various test schemes of IEEE involving different stability indicators as target vectors for training the models.

The calculations, however, show that when security is assessed in real time the most effective algorithm in terms of such criteria as accuracy, robustness, online adaptability and versatility is the algorithm of Proximity Driven Streaming Random Forest (PDSRF) proposed in [9]. Therefore, it is the PDSRF algorithm implemented in the programming language $\mathrm{C}++$, which was used as a basis for the developed automatic intelligent system for a real time security assessment of a power system. Moreover, the combination of the original properties of PDSRF algorithm and capabilities of L-index as a target vector for training a model made it possible to implement the following functions (1) the on-line alarming and predicting of potential dangerous states; (2) control security with localization of "critical" nodes in power systems; (3) interact with relay protection and automation systems.

\section{Proximity Driven Streaming Random Forest ALGORITHM}

In many papers devoted to the studies of the power system security on the basis of decision trees, the authors suggest using the model off-line and, instead of adaptation, periodically update the model $[10,11]$. However, even in the case of retraining, the complete training of the model is associated with additional time, which excludes the retraining in real time. This problem can be solved by on-line methods, which update the existing model, using new data without its total restructuring [11]. A PDSRF algorithm employed in the paper can serve as one of such models.

\section{A. Streaming Classifier Based on Random Forest}

For real power systems learning algorithms are supposed to work in dynamic environments with data continuously generated in the form of a stream on not necessarily equally spaced time intervals. Data stream processing commonly relies on single scans of the training data and implies restrictions on memory and time. Changes caused by dynamic environments (e.g. consumer behaviour in power systems) can be categorised into sudden or gradual concept drift subject to appearance of novel classes in a stream and the rate of changing definitions of classes.

Ensemble methods enable to build a reliable decision rules for feature space classification in the presence of many possible states of the system. Methodologically ensemble approaches allow concept-drift to be handled in the following ways: base classifier adaptation, changing in training dataset $[12,13]$, ensemble aggregation rule changing or changing in structure of an ensemble (pruning or growing). In this paper we employ the PDSRF algorithm which exploit combinations of these approaches.
Contrary to conventional algorithms we use the accuracy weighted ensemble (AWE) method as an aggregation rule of ensemble. This allows us to adapt the entire classifier by changing the weights of the base learners. In order to obtain the classifiers weight estimation we should store samples. For this purpose we use a sliding windows approach which is used in the periodically updated Random Forest [14]. The length of this window is fixed and can be estimated by cross-validation. For the sake of time and memory optimization Extremely randomized trees [15] is used as a base learner instead of original randomized trees.

\section{B. Base Classifier Weighting Function and Forgetting strategy}

Following the AWE approach proposed in [16] we use an error rate to produce weights (1) of classifiers, where $E$ is an new block testing error for $i$-th classifier, is a small parameter

$$
\omega_{i}=\frac{1}{E_{i}^{2}(x)+\Delta},
$$

One of the main problems in concept-drifting learning is to select the proper forgetting strategy and forgetting rate [17]. The classifier should be adaptive enough to handle changes. In this case different strategies can be more appropriate to different types of drift (for example, sudden and gradual drifts). In this paper we focus on gradual changes only. As result, we propose ensemble pruning technique to handle the concept drift. This technique uses the classic replace-the-looser approach [17] to discard trees with high error on new block samples.

\section{Proposed algorithm}

Thus, to predict the sample we propose the following algorithm.

1) we use a stored window to find similar items using the specified similarity metric;

2) we evaluate our current ensemble on similar examples;

3 ) we compute weights adherence to errors on $\mathrm{k}$ similar samples.

On every chunk the algorithm tests all the trees to choose the poorest base learner and replace it with new one trained on new block data. This process is iterative while the ensemble error on new block samples is higher than a specified threshold.

Based on the PDSRF algorithm, we developed an innovative on-line approach to the assessment and control of voltage security of power systems, implemented in the $\mathrm{C}++$ programming language. The main qualitative distinction of this method from the other modern approaches is the PDSRF algorithm capability to independently and adaptively change in real time when serious changes occur in the received telemetry data (generator tripping, sharp increase in load, etc.) without loss of accuracy when identifying the power system operating conditions. In fact, this makes it possible to switch from a simple classifier of the system states to the intelligent detector of abnormalities, i.e. dangerous pre-emergency states. 


\section{The Use of L-INDEX IN The Problem of ONLINE Voltage SECURITY CONTROL}

As said above it is more sensible to use system variables based voltage stability indices of the power system in real time. Therefore, in the study we use L-index, being one of the effective indices from this group, as a target indicator of system stability when training the PDSRF model.

The L-index is proposed by Kessel and Glavitsch in [18] as an indicator of impeding voltage stability. Starting from the subsequent analysis of a power line equivalent model, the authors developed a voltage stability index based on the solution to power flow equations. The $\mathrm{L}$ index is a quantitative measure for the estimation of the distance of the actual state of the system to the stability limit. The L-index describes the stability of the entire system with the expression:

$$
L=\max _{j \in \alpha_{L}}\left(L_{j}\right)
$$

where $\alpha_{L}$ is a set of load nodes. $L_{j}$ is a local indicator that determines the buses which can be sources of collapse. The L-index varies in a range between 0 (no load) and 1 (voltage collapse) and used to provide meaningful voltage instability information during dynamic disturbances in the system.

Kessel and Glavitsch reformulate the local indicator $L_{j}$ in terms of the power as:

$$
L=\left|1+\frac{\dot{U}_{0 j}}{\dot{U}_{j}}\right|=\left|\frac{\dot{S}_{j}^{+}}{\dot{Y}_{j j}^{+*} U_{j}^{2}}\right|=\frac{S_{j}^{+}}{Y_{j j}^{+} U_{j}^{2}} .
$$

where $Y_{j j}^{+}$- transformed admittance; $U_{j}$ - voltage of the load bus $j ; S_{j}^{+}$- transformed complex power, which can be calculated as $\dot{S}_{J}^{+}=\dot{S}_{j}+\left(\sum_{j \in \alpha_{L}, i \neq j} \frac{\dot{Z}_{j i}^{*} \dot{S}_{i}}{\dot{S}_{j j}^{*} \dot{U}_{i}}\right) \dot{U}_{j}, ; \dot{Z}_{j i}^{*}, \dot{Z}_{j j}^{*}$ - off-diagonal elements and leading elements of impedance matrix.

The voltage stability indicators are known to be not only an effective method to assess the system stability but also they underlie the control of electric power system security. In [15] the authors propose a variant of L-index calculation, which was used to develop an L-Q sensitivity analysis method, which eases the quantitative analysis of voltage influence between different nodes

According to the basic differential property of the L-index we devised a common analytical algorithm for reactive power optimization. The algorithm can be used to determine the required reactive power injection for the load node, $\Delta Q$. Based on the applied methodology, a large-scale power system will operate in an optimal steady state under the minimum $L_{\text {sum }}=L_{1}+L_{2}+\ldots+L_{m}$ which represents a sum of local indices $L_{j}$. In this case the function of the first partial derivative is defined as follows:

$$
\frac{\partial L_{\text {sum }}}{\partial \Delta Q}=\left[\begin{array}{c}
\frac{\partial L_{\text {sum }}}{\partial \Delta Q_{1}} \\
\vdots \\
\frac{\partial L_{\text {sum }}}{\partial \Delta Q_{m}}
\end{array}\right]=
$$

$$
=\left[\begin{array}{c}
\sum_{i \in \alpha_{L}, i \neq j}-\frac{1}{U_{j}} \frac{X_{j 1}}{U_{1}} \frac{\mu}{\sqrt{\mu^{2}+\gamma^{2}}} \\
\vdots \\
\sum_{i \in \alpha_{L}, i \neq j}-\frac{1}{U_{j}} \frac{X_{j m}}{U_{m}} \frac{\mu}{\sqrt{\mu^{2}+\gamma^{2}}}
\end{array}\right]=0,
$$

where $\mu=\sum_{i \in \alpha_{L}, i \neq j} \frac{\left(Q_{i}+\Delta Q_{i}\right) X_{j i}}{U_{i}} ; \gamma=\sum_{i \in \alpha_{L}, i \neq j} \frac{-P_{i} X_{j i}}{U_{i}}$.;

The calculations carried out in [19] on different IEEE test systems show that the application of this approach makes it possible to improve the voltage stability by reactive power injections at load nodes. The injections are calculated from the L-index minimization conditions, and keep a system under heavy load conditions away from instability boundaries. Nevertheless, the authors state that despite the relative simplicity of the calculation, this method requires considerable computational efforts and its application in the real time problems can be complicated.

In this paper we suggest supplementing and modifying this approach by using trainable models able to learn to calculate both the global L-index for the security assessment of an entire system, and the required injections $\Delta Q$, when determining the place and magnitude of corrective actions. This will allow us to apply this methodology in real time.

\section{Structure of AN Automatic Intelligent System}

The general structure of the developed system is presented in Fig. 1. The scheme shows that the proposed system consists of two main models on the basis of the PDSRF algorithm local and global.

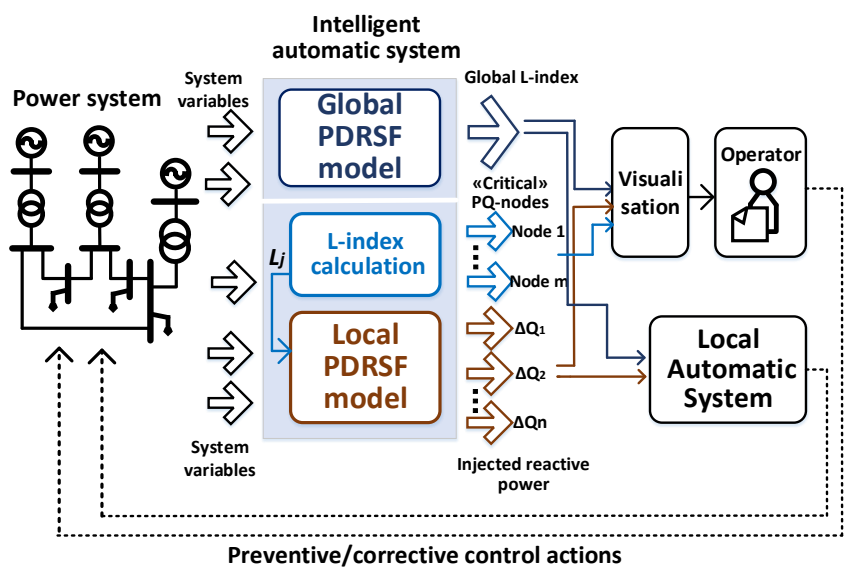

Fig. 1. Structural scheme of an automatic intelligent system for voltage security control

Global model is trained to correctly identify the global Lindex on the basis of system variables of a power system such as voltage at nodes, loads, power flows, etc. Here an output value of the L-index is interpreted as a security signal (indicator) of the entire power system. The local model on the basis of the PDSRF algorithm, in turn, is trained to determine the required reactive power injections $\Delta Q$ for load nodes. The inputs for the local model are represented by the same 
operating parameters and calculated values of the local L-index at a current time instant. Training of the local PDSRF model is based on the equation of partial derivative of expression (4). As a result, the trained model is able to determine the values $\Delta Q$ to perform corrective and/or preventive control actions online. Additionally, the security intelligent system can provide local signals in the form of local L-indices for each load at load node.

In the end, such a structure makes it possible to implement the above functions of on-line alarming, localization and interaction with automatic systems. For example, the system signal on the basis of L-index, which is delivered to operator through the visualization block, informs the dispatcher on the general level of security in the analyzed power system (high, low, emergency), and allows the operator to predict (estimate) the extent to which the current state of the electric power system is dangerous in terms of its closeness to voltage collapse.

In the case of a dangerous state identification (low security) the local signals on the basis of local L-index, formed by local model, enable us to localize critical load nodes at which the system is at its closest to the stability loss. The corrective and/or preventive control actions can be implemented on-line on the basis of the injections $\Delta Q$ generated by the local model based on the PDSRF algorithm for load nodes. Such control actions can both adjust the operating conditions in terms of their optimality according to some economic criteria, or, in the case of a decrease in security, keep the conditions away from the instability boundaries.

It is important to note that the output signals of the alarming system are delivered both to the operator and directly to the operating automation. Interactions with the automation allow us where necessary to correct the actions of agents, since it is they that control the reactive power sources to regulate voltage in order to prevent the development of an emergency process. Operator, in turn, using the recommendations of the intelligent system (in case of a security decrease), can adjust the protective relay settings by decreasing the settings with respect to time, increasing sensitivity of startup signals of the emergency control functions through the selection of an appropriate group of settings, etc. Despite the fact that the proposed structure suggests a certain interaction with dispatcher to control the power system security, we see the main mechanism of the developed intelligent system operation to be mostly automatic, where many control actions are generated with the minimum involvement of the operator.

\section{Test Calculations}

The efficiency of the proposed intelligent system was tested on IEEE 118 test system. The database of possible states of the test system for model training was generated by quasidynamic modeling in the MATLAB environment. At each step of the load increase in the system, emergency events are modeled randomly (Fig. 2). The disturbances included losses of generation and connection of a large consumer at specified nodes. A set of the obtained system states was used to calculate the values of global L-index, and on the basis of local indices $L_{j}$, the reactive power injections $\Delta Q$ were found for each load node. As result, we computed the attribute values and pre-classified based on the L-index the obtained states as normal, alarm, emergency and collapse. These characteristics were applied as class marks for training and testing the models on the basis of the PDSRF algorithm. Global and local PDSRF models are implemented in $\mathrm{C}++$.

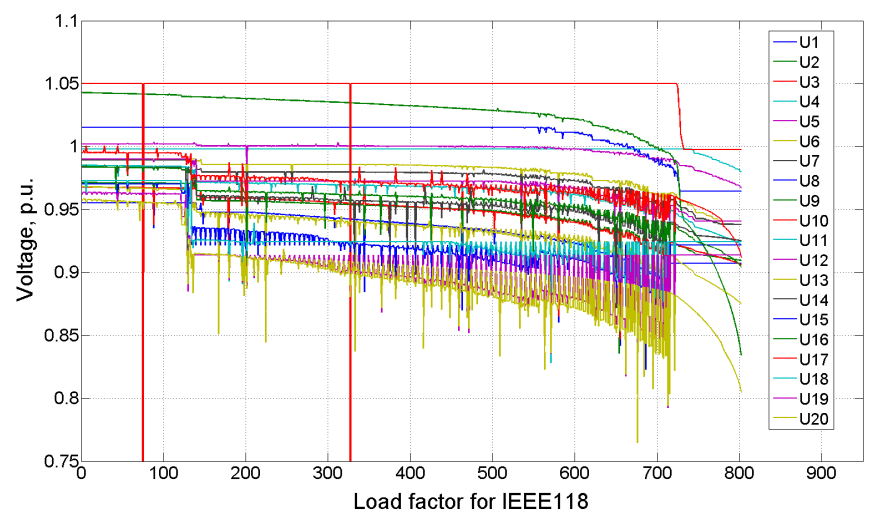

Fig. 2. Changes in voltage profiles of the IEEE 118 system

In order to make simulation data more close to behaviour of real power systems we propose to add sharp change of power load on some buses (Fig. 3). These changes can be considered as a connection of big consumer. Thus the data will contain concept-drift which should be efficiently handled by classification algorithms.

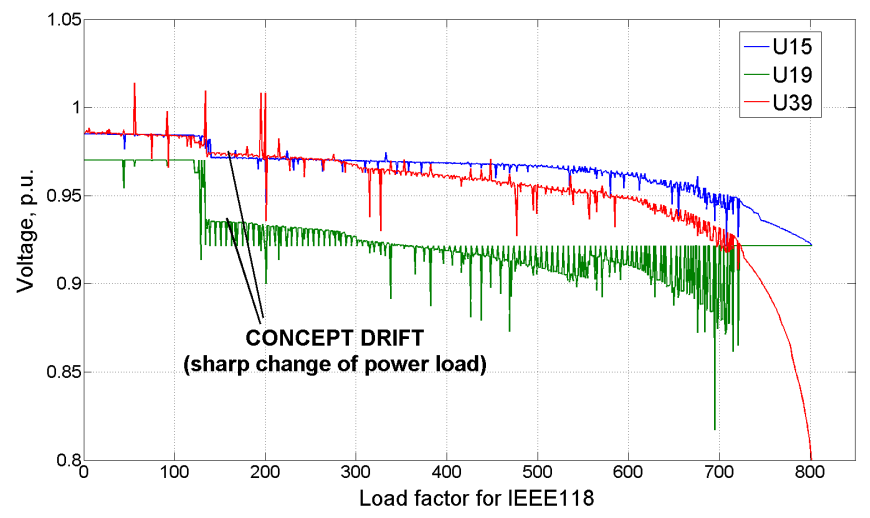

Fig. 3. Voltage profiles at load buses with a concept-drift effect

The simulation results based on computation of local Lindex indicate that buses 103, 105, 106 and 110 are the critical buses (Fig. 4). At this time the bus 105 is the more critical bus for this system. This means that for IEEE 118 system the voltage stability margin is limited by the outage of line 105 .

The testing results of the PDSRF algorithm compared to the other machine learning models are presented in Table 1.

As result, testing of the automatic intelligent system demonstrated that the developed on-line approach on the basis of 


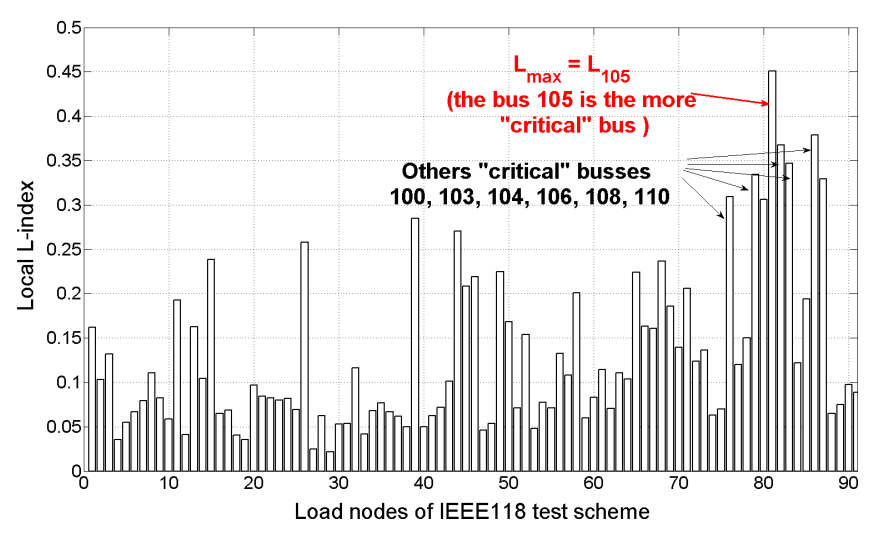

Fig. 4. The local L-index values for one of steady-state condition of IEEE 118

TABLE I

ACCURACY OF VARIOUS MACHINE LEARNING ALGORITHMS ON REPARED DATASET.

\begin{tabular}{lll}
\hline Algorithm & Accuracy, \% & Kappa, \% \\
\hline Global PDSRF model & 97.24 & 95.30 \\
Support Vector Machine & 81.54 & 64.92 \\
Random Forest & 96.01 & 93.24 \\
Gradient Boosting Trees & 93.64 & 89.41 \\
Extreme Learning Machine & 80.80 & 65.08 \\
\hline
\end{tabular}

PDSRF method makes it possible to highly accurately identify the system level of security in the test electric power system. For example, the accuracy of state identification for the scheme IEEE 118 made up $97.24 \%$ (Table 1) which is approximately by $10 \%$ higher than the accuracy of other known intelligent approaches presented in some international peer-reviewed journals over the past years. These approaches include neural networks of Kohonen, supporting vector machines, hybrid neural network models, various algorithms of decision trees. This means that in all the models, except the PDSRF model, the accuracy declines at modeling of significant disturbances in the system, i.e. they could not adapt in real time and required additional training (updating).

Moreover, the calculations show that the proposed on-line approach on the basis of PDSRF provides lower errors (rootmean square error (RMSE) of order $13 \%$ for IEEE 118) and high speed of solving process (about centiseconds for each steady state of IEEE 118 compared to 30-40 minutes in the traditional approach) ${ }^{1}$ when determining the additional reactive power injections (Table 2). This fact makes it possible to effectively apply the automatic intelligent system for monitoring to control security in power systems of large dimension in real time.

The obtained values of additional injections were used for reactive power compensation by using reactive power sources, which decreased $L_{\text {sum }}$, whose increase is indicative of even

\footnotetext{
${ }^{1}$ All calculations were performed on the workstation c Intel processors (R) Core (TM) i7-4930K @ 3.40 GHz 3.30GHz.
}

TABLE II

THE EFFICIENCY OF VARIOUS MACHINE LEARNING ALGORITHMS WHEN DETERMINING THE ADDITIONAL REACTIVE POWER INJECTIONS

\begin{tabular}{lccll}
\hline Algorithm & RMSE & MAE & $\begin{array}{l}\text { Train } \\
\text { time, s }\end{array}$ & $\begin{array}{l}\text { Test } \\
\text { time, s }\end{array}$ \\
\hline Local PDSRF model & 0,1299 & 0,1116 & 4,812 & 0,00149 \\
Support Vector Machine & 0,1498 & 0,1254 & 3,441 & 0,00167 \\
Random Forest & 0,1502 & 0,1271 & 0,811 & 0,00153 \\
Gradient Boosting Trees & 0,1463 & 0,1234 & 4,671 & 0,00282 \\
Extreme Learning Machine & 0,1517 & 0,1282 & 0,021 & 0,00153 \\
\hline
\end{tabular}

greater proximity of voltage collapse, first of all for the heavy load and dangerous conditions of IEEE 118 system (Fig. 5).

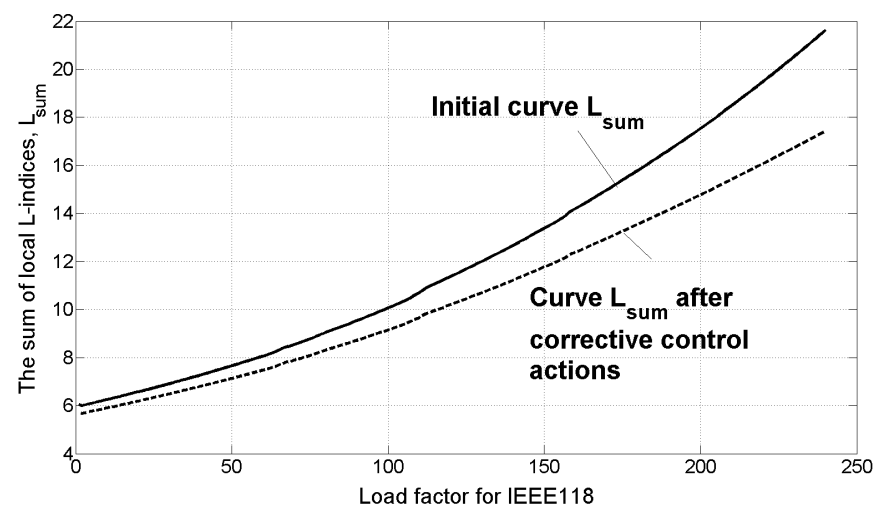

Fig. 5. The curves $L_{\text {sum }}$ before and after corrective control actions

\section{CONCLUSIONS}

We devised an innovative on-line method for the assessment and control of voltage security of power system, using the technology of online decision trees, i.e. PDSRF, implemented in the language $\mathrm{C}++$. The main qualitative distinction of this approach from the other modern approaches is the capability of PDSRF to independently and adaptively change in real time in case of serious changes in the received telemetry data without loss of accuracy while identifying the conditions of electric power system.

This paper presents a PDSRF model for voltage security assessment based on the L-index as security label. This voltage stability index which is based on fundamental Kirchoff-Laws and can reflect the weak point where to locate the vulnerable locations and can predict collapse point of the system. The bus with the highest L-index value will be the most vulnerable bus in the system and hence this method helps in identifying the weak areas in the system which need critical reactive power support.

Voltage stability L-index indicator have been employed for security control using the PDSRF algorithm in order to safely trace the concept drift in data stream and perform the security assessment of the whole system. Experiments with IEEE 118 system various failure scenarios have demonstrated the efficiency of proposed approach. The on-line PDSRF 
method can be to used for improving the voltage stability margin in real time using reactive power sources, generator excitation and on load tap changer transformers as controllers for different loading conditions.

\section{REFERENCES}

[1] IEEE PES CAMS Task Force on Understanding, Prediction, Mitigation and Restoration of Cascading Failures Initial Review of Methods for Cascading Failure Analysis in Electric Power Transmission Systems, In Proc. IEEE PES General Meeting, Pittsburgh, PA USA July 20081.

[2] W. R. Lachs Voltage instability in interconnected power systems: A simulation approach, IEEE Trans. Power Systems, 1992, Vol. 7, No.2.

[3] P. A.Lof, G.Andersson, and D.Hill, "Voltage stability indices for stressed power systems"'. IEEE Trans. on Power Systems, 1993. vol. 8, no.1: p. 326-335.

[4] Danish Mir Sayed Shah. Voltage Stability in Electric Power System: A Practical Introduction.Logos Verlag Berlin GmbH, 2015.

[5] Mahesh Jung Karki Methods for online voltage stability monitoring, $\mathrm{PhD}$ Dissertation, Iowa State University, 2008.

[6] M. Negnevitsky, N. Tomin, V. Kurbatsky, D. Panasetsky, A. Zhukov, Ch. Rehtanz A Random Forest-Based Approach for Voltage Security Monitoring in A Power System, In Proc. IEEE Powertech, Eindhoven, 2015

[7] M. Negnevitsky, N. Voropai, V. Kurbatsky, N. Tomin, and D. Panasetsky, Development of an Intelligent System for Preventing Large-Scale Emergencies in Power Systems, in Proc. IEEE/PES General Meeting, Vancouver, BC, Canada, 2013

[8] A. Zhukov, N. Tomin, D. Sidorov, D. Panasetsky, V. Spirayev A Hybrid Artificial Neural Network for Voltage Security Evaluation in a Power System, in Proc. 2015 5th International Youth Conference on Energy (IYCE), Pisa, Italy

[9] A.V. Zhukov, D.N. Sidorov and A.M. Foley Random Forest Based Approach for Concept Drift Handling, Communications in Computer and Information Science J. 2017, vol. 661, pp. 1-9.

[10] R. Diao, AZ Tempe, Kai Sun, V. Vittal, R.J. O’Keefe, M.R. Richardson, N. Bhatt, D. Stradford, S.K. Sarawgi. Decision Tree-Based Online Voltage Security Assessment Using PMU Measurements. IEEE Trans. on Power Systems, Vol. 24, No. 2, May 2009, pp. 832-839.

[11] M. Beiraghi, A.M. Ranjbar Online voltage security assessment based on wide-area measurements, IEEE Trans. on Power Delivery, 2013. Vol. 28, no. 2, pp. 989997.

[12] L. Breiman, Bagging predictors, Machine Learning, vol. 24, no. 2, pp. $123140,1996$.

[13] T. K. Ho, The random subspace method for constructing decision forests, Pattern Analysis and Machine Intelligence, IEEE Transactions on, vol. 20, pp. 832844, Aug 1998

[14] N. Tomin, A. Zhukov, D. Sidorov, V. Kurbatsky, D. Panasetsky, and V. Spiryaev, Random forest based model for preventing large-scale emergencies in power systems, International Journal of Artificial Intelligence, vol. 13, no. 1, pp. 211228, 2015.

[15] P. Geurts, D. Ernst, and L. Wehenkel, Extremely randomized trees, Machine learning, vol. 63, no. 1, pp. 342, 2006.

[16] H. Wang, W. Fan, P. S Yu, J. H. Mining concept-drifting data streams using ensemble classifiers, In Proc. the ninth ACM SIGKDD international conference on knowledge discovery and data mining, 2003, pp. 226235.

[17] L. Kuncheva, Classifier ensembles for changing environment, in Multiple Classifier Systems, 2004 5th Intl. Workshop on (F. Roli, J. Kittler, and T. Windeatt, eds.), pp. 115, Springer-Verlag, 2004.

[18] P. Kessel and H. Glavitsch, Estimating the voltage stability of a power system, IEEE Transactions on Power Delivery, vol. PWRD-1, 1986, no. 3, pp. 346-353

[19] X. Gong, B. Zhang, B. Kong, A. Zhang, H. Li, W. Fang Research on the Method of Calculating Node Injected Reactive Power Based on L Indicator, Journal of Power and Energy Engineering, 2014, vol. 2, pp. 361-367 